Amanda TaVares Pinherro'

Roberto Antonio AraúJo Costa ${ }^{2}$

Joelcio Francisco AbBade ${ }^{3}$

Cláudia Garcia Magalhães ${ }^{4}$

Gláucia Maria Ferreira da Silva Mazeto 5

\title{
Hipertireoidismo na gravidez: repercussões materno-fetais
}

\author{
Hyperthyroidism during pregnancy: maternal-fetal outcomes
}

Artigos originais

Palavras-chave

Complicações na gravidez Glândula tireóide/patologia

Hipertireoidismo

Tireotoxicose

Keywords

Pregnancy complications Thyroid gland/pathology Hyperthyroidism Thyrotoxicosis

\section{Resumo}

OBJETIVO: avaliar a experiência do Hospital das Clínicas da Faculdade de Medicina de Botucatu da Universidade Estadual Paulista "Júlio de Mesquita Filho", no acompanhamento de gestantes com hipertireoidismo. MÉTODOS: foram estudadas, retrospectivamente, 60 pacientes, divididas em grupos com hipertireoidismo compensado (GHC=24) e com hipertireoidismo descompensado $(\mathrm{GHD}=36)$ e comparadas quanto a características clínico-laboratoriais e intercorrências. Para análise dos resultados, foram utilizados o teste $t$ de Student, as tabelas de contingência, regressão linear múltipla e regressão logística múltipla, com nível de significância de 5,0\%. RESULTADOS: propiltiouracil (PTU) foi usado por $94,0 \%$ do GHD e 42,0\% do GHC (p<0,000 1); complicações maternas próximas ao parto ocorreram em 20,6\% do GHD e 1 1,8\% do GHC, sendo que o GHD apresentou três óbitos fetais. Influenciaram nestes: idade materna, nível de T4L (nT4L) e dose de PTU (dPTU) mais elevados, no terceiro trimestre ( $\mathrm{p}=0,007$ ); restrição de crescimento intra-uterino, influenciada por nT4L e dPTU do terceiro trimestre, ocorreu em nove casos do GHD e três do GHC, e oligoâmnio ocorreu em 12 pacientes $(83,3 \%$ do GHD, 16,7\% do GHC), influenciado por idade e nT4L do terceiro trimestre

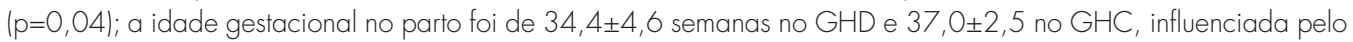
$n T 4 L$ do terceiro trimestre $(p<0,05)$. CONCLUSÕES: $\circ$ GHD apresentou resultados menos satisfatórios que o GHC, influenciados por nT4L e dPTU elevados no terceiro trimestre e por idade mais avançada de algumas gestantes.

\section{Abstract}

PURPOSE: to evaluate the experience of Hospital das Clínicas da Faculdade de Medicina de Botucatu da Universidade Estadual Paulista "Júlio de Mesquita Filho", in the follow-up of pregnant women with hyperthyroidism. METHODS: Sixty patients, divided in groups with compensated hyperthyroidism $(\mathrm{CHG}=24)$ and with uncompensated hyperthyroidism $(U H G=36)$ were retrospectively studied and compared concerning clinical-laboratorial characteristics and intercurrences. The t-Student test, contingency tables, multiple linear regression and multiple logistic regression with significance level at $5.0 \%$ were used. RESULTS: propylthiouracil (PTU) was used by $94.0 \%$ of UHG and by $42.0 \%$ of $\mathrm{CHG}(\mathrm{p}<0.0001)$; maternal complications close to delivery have occurred in $20.6 \%$ of UHG and in $11.8 \%$ of CHG, and UHG presented three fetal deaths, influenced by the mother age, higher level of T4L (IT4L) and of PTU dose (PTUd) in the third trimester $(p=0.007)$; restriction of intra-uterine growth, influenced by IT4L and PTUd in the third trimester has occurred in nine UHG and in three CHG cases, and oligoamnios has occurred in 12 patients $183.3 \%$ of UGH and $16.7 \%$ of $\mathrm{CGH})$, influenced by age and IT4L in the third trimester $(p=0.04)$; the gestational age at delivery was $34.4 \pm 4.6$ weeks in UHG and $37.0 \pm 2.5$ in CHG, influenced by the T4LI in the third trimester ( $p<0.05)$. CONCLUSIONS: the UHG has presented less satisfactory results than CHG, influenced by high IT4L and PTUd in the third trimester, and by more advanced age of some pregnant women.
Correspondência:

Gláucia Maria Ferreira da Silva Mazeto Departamento de Clínica Médica da Faculdade de Medicina de Botucatu da Universidade Estadual Paulista "Júlio de Mesquita

Filho" - UNESP

CEP 18618-000 - Botucatu/SP E-mail: gmazeto@fmb.unesp.br

Recebido

$9 / 6 / 08$

Aceito com modificacões

$10 / 9 / 08$
Departamentos de Ginecologia e Obstetrícia e de Clínica Médica da Faculdade de Medicina de Botucatu da Universidade Estadual Paulista "Júlio de Mesquita Filho" - UNESP - Botucatu (SP), Brasil.

' Residente do Departamento de Pediatria da Faculdade de Medicina de Botucatu da Universidade Estadual Paulista "Júlio de Mesquita Filho" - UNESP - Botucatu (SP), Brasil.

2 Doutor, Professor-assistente e Coordenador do Ambulatório de Gravidez e Tireopatias do Departamento de Ginecologia e Obstetrícia da Faculdade de Medicina de Botucatu da Universidade Estadual Paulista "Júlio de Mesquita Filho" - UNESP - Botucatu (SP), Brasil.

${ }^{3}$ Doutor e Professor-assistente do Departamento de Ginecologia e Obstetrícia da Faculdade de Medicina de Botucatu da Universidade Estadual Paulista "Júlio de Mesquita Filho" - UNESP - Botucatu (SP), Brasil.

${ }^{4}$ Médica do Departamento de Ginecologia e Obstetrícia da Faculdade de Medicina de Botucatu da Universidade Estadual Paulista "Júlio de Mesquita Filho" - UNESP - Botucatu (SP), Brasil.

${ }_{5}^{5}$ Doutora, Professora-assistente e Coordenadora do Ambulatório de Tireopatias do Departamento de Clínica Médica da Faculdade de Medicina de Botucatu da Universidade Estadual Paulista "Júlio de Mesquita Filho" - UNESP - Botucatu (SP), Brasil.

Apoio Financeiro: bolsa de iniciação científica da Fundação de Amparo à Pesquisa do Estado de São Paulo (FAPESP), sob processo $n^{\circ} .05 / 52557-2$ 


\section{Introdução}

O hipertireoidismo complica cerca de 0,1 a $0,4 \%$ das gestações, com a maioria dos casos sendo devidos à doença de Graves (DG) ${ }^{1}$. Outras causas, próprias da gestação, tais como a doença trofoblástica gestacional e tireotoxicose gestacional transitória, podem também levar ao excesso de hormônios tireóideos circulantes ${ }^{2,3}$. Dependendo da etiologia da tireotoxicose, esta pode variar de branda e transitória a grave e duradoura. A DG, por exemplo, apesar de levar a uma tireotoxicose mais intensa, pode melhorar progressivamente ao longo da gestação, devido a uma maior tolerância imunológica ${ }^{4,5}$.

Quando o hipertireoidismo não regride com a evolução da gestação, comprometendo, particularmente, o segundo e terceiro trimestres, várias complicações maternas e fetais são referidas ${ }^{1}$. Entre estas complicações, podem ser citadas maiores incidências de eclâmpsia, insuficiência cardíaca congestiva, edema agudo de pulmão, arritmias cardíacas e crise tireotóxica. Além disso, também têm sido relatadas maiores taxas de abortamento, natimortalidade, prematuridade, baixo peso ao nascer e malformações fetais ${ }^{6}$.

O diagnóstico e tratamento precoces e adequados parecem ser essenciais para a prevenção destas complicações ${ }^{6}$, visando-se manter os níveis de tiroxina livre no limite superior da normalidade, buscando-se o eutireoidismo clínico materno, com o uso da menor dose possível de medicação antitireoidiana ${ }^{1,6-9}$.

A relativa dificuldade em engravidar, experimentada pelas pacientes com tireotoxicose manifesta, dificulta a realização de estudos sobre o tema, particularmente no âmbito nacional ${ }^{1}$. O objetivo deste estudo foi avaliar a influência do hipertireoidismo descompensado sobre a morbidade materno-fetal, em um grupo de gestantes acometidas pelo distúrbio.

\section{Métodos}

Este estudo foi aprovado pelo Comitê de Ética em Pesquisa da Faculdade de Medicina de Botucatu da Universidade Estadual Paulista "Júlio de Mesquita Filho" (UNESP) e realizado de forma retrospectiva, observacional, descritiva e comparativa.

Foram revistos os prontuários médicos de 237 gestantes tireopatas, acompanhadas no Ambulatório de Tireopatias e Gravidez do Hospital das Clínicas da Faculdade de Medicina de Botucatu da UNESP, entre os anos de 1997 e 2005. Destes prontuários, foram selecionados 60 casos $(25,4 \%)$ que apresentavam tireotoxicose endógena durante a gravidez, tendo sido avaliada uma gestação por paciente. Estas não apresentavam acompanhamento prévio na instituição sede do estudo, e os dados a respeito de gestações ou seguimento médico anteriores não estavam disponíveis.
Os casos foram divididos em dois grupos: um com gestantes com hipertireoidismo compensado (GHC) e outro com hipertireoidismo descompensado (GHD). Para inclusão nos grupos, o critério utilizado foi o resultado da dosagem do nível sérico de tiroxina livre (T4L), apresentado por cada paciente durante todo o período, compreendendo o segundo e terceiro trimestres e o momento do parto. Os casos com níveis hormonais dentro da faixa de referência normal foram classificados como GHC e os com níveis acima da mesma foram classificados como GHD.

Para descrição geral dos grupos, foram avaliadas as seguintes características maternas: idade, tabagismo, idade gestacional no início do seguimento e número de consultas de pré-natal. Para estudo dos grupos, quanto a características relativas à tireotoxicose, foram avaliados: etiologia da doença, tempo presumido de doença (em meses), presença de sinais e sintomas de tireotoxicose, níveis de T4L, tirotrofina (TSH), anticorpos antitireoperoxidase (antiTPO) e anti-receptor do TSH (TRAb), doses de drogas antitireoidianas (DAT), nos diferentes trimestres, e uso de propranolol. Para averiguarem-se as repercussões materno-fetais da tireotoxicose descompensada, os grupos foram comparados quanto a: idade gestacional no parto (em semanas), tipo de parto, complicações materno-fetais (pré-eclâmpsia, descolamento prematuro de placenta, prematuridade, oligoâmnio, restrição de crescimento intra-uterino (RCIU)), ocorridas durante a gestação. Foi também avaliada a possível relação entre estes parâmetros e a idade materna, etiologia da tireotoxicose, níveis de T4L e TRAb e doses de DAT, nos trimestres.

A etiologia da tireotoxicose foi considerada auto-imune, quando associada à oftalmopatia e/ou à positividade dos auto-anticorpos TRAb ou antiTPO no soro, ou devido a outras causas, quando não associada.

As dosagens de T4L, TSH e antiTPO foram realizadas pelo método da quimioluminescência (DPC, Los Angeles, CA, USA), com valores de referência de 0,8 a $1,9 \mathrm{ng} / \mathrm{dL}$, 0,4 a $4,0 \mathrm{mUI} / \mathrm{mL}$ e $<35,0 \mathrm{UI} / \mathrm{mL}$, respectivamente. As dosagens de TRAb foram realizadas por radioimunoensaio (CIS-Bio International, França), sendo considerados positivos valores maiores do que 11,0 U/L.

Os dados foram tabulados na planilha Excel (Microsoft Corporation $^{\circledR}$, EUA) e submetidos à análise estatística por meio do programa computacional Statistical Package for Social Sciences (SPSS), para Windows (versão 10.0.7). Aplicou-se o teste $t$ de Student para comparação dos parâmetros: idade das pacientes, idade gestacional inicial, número de consultas de pré-natal, idade gestacional no parto, T4L, TSH, TRAb, tempo presumido de doença e dose de propiltiouracil (PTU); utilizaram-se as tabelas de contingência para a comparação dos parâmetros: tabagismo, etiologia auto-imune, sinais e sintomas de tireotoxicose e uso de DAT e propranolol; para estudar a interferência 
de variáveis quantitativas entre si, foi usada a regressão linear múltipla; para avaliar a interferência entre parâmetros qualitativos e quantitativos, e suas respectivas interações, foi utilizada a regressão logística múltipla e o nível de significância adotado foi 5,0\% .

\section{Resultados}

Trinta e seis gestantes foram selecionadas para o GHD e 24 para o GHC. As características gerais e as relativas à tireotoxicose, de ambos os grupos, podem ser observadas na Tabela 1. Já os níveis de T4L, TSH, TRAb e antiTPO, de acordo com o trimestre gestacional, encontram-se na Tabela 2.

Dezoito pacientes do GHC e 35 do GHD apresentaram etiologia auto-imune (Tabela 1 ), enquanto que seis pacientes do GHC $(25,0 \%)$ e uma do GHD $(2,8 \%)$ apresentaram tireotoxicose por outras causas.

Tabela 1 - Medidas descritivas das características gerais e relativas à tireotoxicose, das pacientes gestantes com hipertireoidismo

\begin{tabular}{|c|c|c|}
\hline \multirow[b]{2}{*}{ Característica } & \multicolumn{2}{|c|}{ Grupos } \\
\hline & $\begin{array}{l}\text { Hipertireoidismo } \\
\text { compensado }\end{array}$ & $\begin{array}{l}\text { Hipertireoidismo } \\
\text { descompensado }\end{array}$ \\
\hline$n(\%)$ & $24(40)$ & $36(60)$ \\
\hline Idade* (anos) & $25,0 \pm 6,0$ & $26,0 \pm 5,0$ \\
\hline Idade gestacional inicial* (semanas) & $17,0 \pm 9,0$ & $17,0 \pm 6,0$ \\
\hline Presença de tabagismo (\%) & 18,2 & 27,0 \\
\hline Número de consultas de pré-natal* & $5,0 \pm 2,0$ & $6,0 \pm 2,0$ \\
\hline Etiologia auto-imune (\%) & 75,0 & $97,2^{\dagger}$ \\
\hline $\begin{array}{l}\text { Tempo presumido de doença* } \\
\text { (meses) }\end{array}$ & $40,9 \pm 34,3$ & $59,1 \pm 213,4$ \\
\hline Presença de sintomas (\%) & 62,5 & $88,9^{\dagger}$ \\
\hline Presença com sinais (\%) & 83,3 & 94,4 \\
\hline $\begin{array}{l}\text { Níveis de T4L durante a gestação* } \\
\text { (ng/dL) }\end{array}$ & $1,64 \pm 0,9$ & $3,2 \pm 1,7^{\#}$ \\
\hline $\begin{array}{l}\text { Níveis de TSH durante a gestação* } \\
(\mathrm{mUl} / \mathrm{mL})\end{array}$ & $0,33 \pm 0,40$ & $0,04 \pm 0,06^{*}$ \\
\hline Uso de propiltiouracil (\%) & 42,0 & $94,0^{\#}$ \\
\hline Uso de propranolol (\%) & 20,8 & 58,3 \\
\hline
\end{tabular}

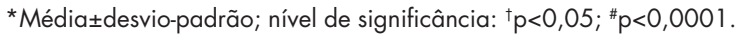

As DAT foram utilizadas em 45 pacientes $(75,0 \%)$ : uma recebeu metimazol e 44 receberam PTU. Além disso, 15 gestantes apresentaram hipertireoidismo subclínico e não receberam DAT. Comparando-se as pacientes tratadas com DAT nos grupos GHC e GHD, observou-se uma maior porcentagem no GHD (Tabela 1). As doses de PTU variaram de 50 a $800 \mathrm{mg} / \mathrm{dia}$, sendo que, no primeiro trimestre, apenas duas pacientes $(4,4 \%)$ recebiam doses maiores que $300 \mathrm{mg} / \mathrm{dia}$, enquanto que, no terceiro trimestre, $25(55,6 \%)$ as utilizavam. O GHD apresentou maior porcentagem de gestantes com doses superiores a $300 \mathrm{mg} / \mathrm{dia}$, no segundo e terceiro trimestres (Tabela 2).

Vinte e uma gestantes do GHD e cinco do GHC utilizaram propranolol em alguma fase da gravidez (Tabela 1). Este uso foi esporádico, em situações de maior descompensação clínica, quando as freqüências cardíacas atingiam 120 ou mais batimentos por minuto, normalmente não ultrapassando doses de $80 \mathrm{mg} /$ dia e períodos de 30 dias.

Complicações maternas, durante todo o período gestacional, ocorreram em 26 pacientes $(43,3 \%)$, conforme Tabela 3. Destas, 65\% eram do GHD e 35\% do GHC e as complicações foram: hiperemese, pré-eclâmpsia, eclâmpsia, diabetes gestacional, crise tireotóxica e insuficiência cardíaca. Complicações mais próximas ao momento do parto, tais como rotura prematura de membranas e pré-eclâmpsia, ocorreram em $20,6 \%$ das pacientes do GHD e em 11,8\% das do GHC (diferença não significante), influenciadas pela idade das gestantes, nível de T4L e dose de PTU mais elevados, no terceiro trimestre $(\mathrm{p}=0,007)$.

Complicações fetais como RCIU, oligoâmnio e óbito fetal foram diagnosticados, durante o seguimento prénatal, em 16 das pacientes do GHD e em cinco das do GHC (Tabela 3). Doze gestantes apresentaram fetos com RCIU: nove $(75,0 \%)$ do GHD e três $(25,0 \%)$ do GHC. Destas 12 , quatro $(33,3 \%)$ fizeram uso de propranolol em alguma fase da gestação. A associação de níveis de T4L e doses de PTU mais elevados, no terceiro trimestre,

Tabela 2 - Medidas descritivas de parâmetros laboratoriais e medicamentosos das pacientes gestantes com hipertireoidismo compensado (GHC) ou descompensado (GHD), de acordo com o trimestre da gravidez

\begin{tabular}{|c|c|c|c|c|c|c|c|c|c|}
\hline \multirow{2}{*}{ Parâmetro } & \multicolumn{2}{|c|}{ Primeiro trimestre } & \multirow[b]{2}{*}{$\mathbf{p}$} & \multicolumn{2}{|c|}{ Segundo trimestre } & \multirow[b]{2}{*}{$\mathbf{p}$} & \multicolumn{2}{|c|}{ Terceiro trimestre } & \multirow[b]{2}{*}{$\mathbf{p}$} \\
\hline & GHC & GHD & & GHC & GHD & & GHC & GHD & \\
\hline T4L* (ng/dL) & $2,2 \pm 0,6$ & $4,0 \pm 1,3$ & 0,006 & $1,2 \pm 0,3$ & $4,1 \pm 2,1$ & 0,000 & $1,2 \pm 0,2$ & $2,9 \pm 1,7$ & 0,000 \\
\hline $\mathrm{TSH}^{*}(\mathrm{mUI} / \mathrm{mL})$ & $0,23 \pm 0,44$ & $0,01 \pm 0,00$ & 0,193 & $0,46 \pm 0,51$ & $0,03 \pm 0,05$ & 0,000 & $0,32 \pm 0,39$ & $0,05 \pm 0,12$ & 0,000 \\
\hline TRAb* (U/L) & $39,0 \pm 50,0$ & $254,0 \pm 329,0$ & 0,156 & $27,0 \pm 50,0$ & $112,0 \pm 136,0$ & 0,04 & $15,0 \pm 23,0$ & $72,0 \pm 99,0$ & 0,04 \\
\hline TRAb+ (\% casos) & $\mathrm{NE}^{\ddagger}$ & $\mathrm{NE}^{\ddagger}$ & $\mathrm{NE}^{\ddagger}$ & 38,5 & 85,0 & 0,008 & 26,7 & 85,0 & 0,001 \\
\hline AntiTPO* (UI/mL) & $500,0 \pm 478,0$ & $736,0 \pm 408,0$ & 0,401 & $168,0 \pm 206,0$ & $330,0 \pm 359,0$ & 0,198 & $74,0 \pm 104,0$ & $201,0 \pm 290,0$ & 0,087 \\
\hline
\end{tabular}

*Média \pm desvio-padrão; $\mathrm{PTU}^{\dagger}=$ propiltiouracil; $\mathrm{NE}^{\ddagger}=$ não estudado. 
Tabela 3 - Medidas descritivas de parâmetros relacionados às repercussões materno-fetais do hipertireoidismo em gestantes

\begin{tabular}{|c|c|c|}
\hline Parâmetro & \multicolumn{2}{|c|}{ Grupos } \\
\hline Complicações maternas (\%) & 37,5 & 47,2 \\
\hline Idade gestacional no parto* (semanas) & $37,0 \pm 2,5$ & $34,4 \pm 4,6^{\dagger}$ \\
\hline Parto cesáreo (\%) & 35,5 & 54,5 \\
\hline
\end{tabular}

Média \pm desvio-padrão; nível de significância: ${ }^{\dagger} p<0,05$.

relacionou-se com a presença de RCIU ( $\mathrm{p}=0,04)$. Em relação ao oligoâmnio, das 12 pacientes que o apresentaram, $83,3 \%$ eram do GHD e 16,7\% eram do GHC. A idade das pacientes e o nível de T4L mais elevados, no terceiro trimestre, relacionaram-se com a presença desta complicação ( $\mathrm{p}=0,04)$. Os três únicos óbitos fetais ocorreram no GHD e se relacionaram com a interação entre idade das pacientes, nível de T4L e dose de PTU mais elevados, no terceiro trimestre $(\mathrm{p}=0,007)$.

A idade gestacional no parto foi menor no GHD do que no GHC (Tabela 3). Esta se relacionou com o nível de T4L do terceiro trimestre, sendo que, quanto maior o nível do hormônio, menor a idade gestacional $(\mathrm{p}<0,05)$. Partos prematuros ocorreram em 54,3\% dos casos, no GHD, e em 47,1\%, no GHC ( $\mathrm{p}=0,42)$. Analisando-se o número de prematuros, $70,3 \%$ eram do GHD, enquanto 29,6\% eram do GHC.

Porcentagens expressivas de gestantes dos dois grupos foram submetidas à cesárea (Tabela 3) e as principais indicações para este tipo de parto foram: sofrimento fetal agudo e crônico, descolamento prematuro de placenta, número de cesáreas anteriores, desproporção cefalopélvica e apresentação pélvica.

\section{Discussão}

A freqüência de tireotoxicose relatada na gestação é de 0,05 a 2,9\% $6,7,9-12$. Esta grande variação se deve, provavelmente, às diferenças regionais na prevalência das principais causas, às pesquisas realizadas em vários tipos de serviços, alguns referenciais e outros não, e aos diferentes graus de busca ativa. No presente estudo, analisando-se o total de gestações do Serviço de Obstetrícia do Hospital das Clínicas da Faculdade de Medicina de Botucatu da UNESP, no período, esta porcentagem foi mais elevada $(0,6 \%)$, provavelmente refletindo a natureza do hospital em questão como centro de referência. Porém, encontra-se bem abaixo da relatada por Rodrigues et al. ${ }^{13}(5,5 \%)$, avaliando uma população de gestantes acompanhadas em um serviço de pré-natal, na cidade de São Paulo.

A etiologia auto-imune foi a causa mais freqüente de tireotoxicose em ambos os grupos, GHC e GHD, embora a porcentagem de pacientes com este diagnóstico tenha sido mais exuberante no segundo. Esta categoria etiológica incluía um número considerável de casos de DG, situação em que a tireotoxicose costuma ser mais grave do que nas demais, o que poderia ter influenciado no quadro mais grave apresentado por este grupo. Embora a DG seja descrita como a causa mais freqüente de hipertireoidismo na gestação, ela é de mais fácil controle medicamentoso do que foi observado ${ }^{2,14}$.

Quanto ao uso de medicação, recomenda-se, como primeira escolha, o PTU, nas menores doses capazes de manter o T4L materno no limite superior da normalidade para pacientes não-grávidas ${ }^{15,16}$. No presente estudo, mais pacientes do GHD utilizaram a medicação em relação ao GHC. Ao se estudarem as doses de PTU utilizadas pelas pacientes, nota-se que elas aumentaram do primeiro para o terceiro trimestres. Embora isto possa sugerir uma piora da tireotoxicose com a evolução da gestação, reflete, na realidade, o uso de um tratamento mais adequado durante o acompanhamento, comparado ao inexistente ou insuficiente, no início da gravidez. As gestantes do GHD foram as que utilizaram maiores doses, sendo que estas se mantiveram elevadas no decorrer dos três trimestres. Pode-se especular que o declínio dos níveis de T4L, principalmente no GHD, deu-se também por estas dosagens elevadas, e não somente pela relatada melhora da auto-imunidade que acontece na gestação ${ }^{4,5}$. Por outro lado, as mais altas doses de PTU no GHD só comprovam a maior intensidade da tireotoxicose neste grupo. Estes dados diferem em muito da literatura, que refere, usualmente, à necessidade doses menores de medicamentos devido à melhora da tireotoxicose que ocorre com a progressão da gestação. $\mathrm{O}$ uso destas doses mais baixas ajudaria, inclusive, a evitar complicações para a criança após o parto ${ }^{17-19}$. Contudo, a literatura concorda que o hipertireoidismo deva ser controlado, mesmo que sejam necessárias doses elevadas de DAT, com o objetivo de se evitar maiores complicações materno-fetais ${ }^{1}$. Assim, analisando-se as pacientes do GHD, doses consideráveis de PTU foram utilizadas para o controle da tireotoxicose.

Uma porcentagem expressiva de casos $(58,3 \%)$ recebeu propranolol, medicamento que tem sido utilizado 
para tratar o hipertireoidismo descompensado, embora seu uso, no final da gestação e por tempo prolongado, tenha sido associado a leves e transitórias hipoglicemia, apnéia e bradicardia neonatais, além de RCIU ${ }^{16}$. No presente estudo, não foram encontrados relatos das primeiras, tendo sido observado, no entanto, casos de RCIU, que por sua vez, têm sido associado ao uso continuado de propranolol ${ }^{6}$. Dos casos que apresentaram esta complicação, apenas $33,3 \%$ haviam feito uso do beta-bloqueador em alguma fase da gestação, e por curto período de tempo. De qualquer forma, apesar da utilização moderada, não se pode excluir a possibilidade de que o propranolol tenha influenciado negativamente no desfecho das gestações.

As complicações maternas, durante a gestação, foram comumente referidas pela literatura ${ }^{16}$ e ocorreram em porcentagens semelhantes nos dois grupos. Este achado difere da maioria dos estudos, os quais relatam uma menor taxa de complicações nas pacientes compensadas do que nas descompensadas ${ }^{16}$, porém, estes trabalhos, em sua maioria, compararam grupos tratados com não tratados. No presente estudo, ambos os grupos acabaram por receber tratamento, ainda que insuficiente, sendo o critério para classificação não a terapia, mas a descompensação do quadro laboratorial. De fato, o nível acima do normal do T4L parece ser o principal fator relacionado ao aumento de complicações maternas. Casey et al. ${ }^{20}$, avaliando 433 gestantes com hipertireoidismo subclínico, não encontraram aumento das taxas de complicações maternas. Considerando-se as pacientes que apresentaram complicações, uma porcentagem expressiva era do GHD, podendo assim observar uma influência da idade das pacientes do nível de T4L e da dose de PTU mais elevados, no terceiro trimestre. A relação entre duração e intensidade do hipertireoidismo com a ocorrência de complicações maternas é amplamente difundida ${ }^{16}$. Porém, neste estudo, como idade e dose de PTU exerceram um efeito conjunto, fica difícil confirmar o papel isolado do nível de T4L. Não foram encontradas referências associando a dose de PTU a estas complicações.

As complicações fetais mais importantes apresentadas na literatura são RCIU, óbito e bócio fetais, geralmente associadas ao descontrole da tireotoxicose ${ }^{1,9}$. No presente estudo, também foram observados casos de RCIU e óbito fetais, não tendo sido referido bócio nas crianças. Ao se analisarem os casos de RCIU, 75,0\% pertenciam ao GHD, sendo que esta complicação apresentou interação com maiores doses de PTU e os níveis mais elevados de T4L, no terceiro trimestre. Vários estudos têm relatado associação entre hipertireoidismo mal controlado, particularmente no terceiro trimestre, e RCIU ou baixo peso ao nascimento ${ }^{16}$, sendo que os níveis maternos de T4L parecem ser o principal fator a influenciar na ocorrência de complicações fetais e neonatais. Casey et al. ${ }^{20}$, avaliando 433 gestantes com hipertireoidismo subclínico, não relataram aumento da prevalência de baixo peso, óbito ou qualquer outra complicação para o concepto. No presente estudo, como as pacientes com níveis mais elevados de T4L provavelmente foram as que receberam as maiores doses de PTU, fica difícil estabelecer se houve ou não um único fator responsável direto por esta complicação. Foram relatados ainda casos de oligoâmnio e, das pacientes que o apresentaram, 83,3\% eram do GHD. Apesar de não terem sido encontradas referências sobre a associação desta complicação com quadros de tireotoxicose, este achado alerta para a possibilidade de relação entre ambos. Esta observação encontra respaldo ao considerar-se a estreita relação existente entre oligoâmnio e RCIU ${ }^{21}$, também freqüentemente observada no GHD. A maior idade das pacientes e os elevados níveis de $\mathrm{T} 4 \mathrm{~L}$, no terceiro trimestre, foram os quesitos que influenciaram no aparecimento desta complicação, não sendo possível a demonstração de um único fator isolado como interferente na presença de oligoâmnio. Contudo, neste caso em particular, fica mais clara a influência do grau de tireotoxicose, no final da gestação, obviamente exacerbada pela faixa etária mais avançada das pacientes. Os três únicos óbitos fetais ocorreram no GHD e foram influenciados pela idade das pacientes, pela dose de PTU e, novamente, pelos níveis mais elevados de T4L, no terceiro trimestre. Este achado, mais uma vez, evidencia a influência negativa que a tireotoxicose mais grave e a faixa etária mais elevada exercem sobre mãe e filho. Apesar de a associação entre hipertireoidismo materno não tratado e abortamento ser relatada, sugerindo que os altos níveis hormonais da mãe causariam hipertireoidismo no feto, este assunto ainda é sujeito a controvérsias ${ }^{16}$. Infelizmente, neste estudo, não foi possível estabelecer um fator único a influenciar neste desfecho. Não foram encontradas referências associando a dose de PTU às complicações fetais citadas.

A literatura refere que o hipertireoidismo não tratado acarreta danos à gestante tanto durante o período gestacional quanto no momento do parto ${ }^{1,5,9,16,22}$. Por outro lado, Idris et al. ${ }^{15}$, analisando um grupo de gestantes com hipertireoidismo tratado, não encontraram aumento da incidência de natimortos, pré-eclâmpsia, anomalias congênitas ou complicações pós-parto. Aqueles autores imputam estes bons resultados ao cuidado antenatal ao qual as pacientes têm acesso e à fidedignidade dos registros sobre suas doenças da tireóide. Analisando-se, de forma geral, a gestação nos dois grupos, verificou-se que a freqüência de complicações foi mais expressiva, embora não estatisticamente significante, no grupo das pacientes com GHD, mesmo 
que elas tenham recebido tratamento; talvez este tenha sido ainda insuficiente. O início tardio do acompanhamento pode ter interferido no desfecho das gestações, uma vez que serviços com melhores resultados referem início de seguimento mais precoce. Em um estudo com 40 gestantes com hipertireoidismo, $85 \%$ iniciaram o seguimento ainda no primeiro trimestre ${ }^{15}$. Por outro lado, considerando-se tanto as complicações maternas quanto as fetais, como o GHD, além de apresentar hipertireoidismo mais exuberante, também recebeu doses mais elevadas de medicações e, algumas vezes, não foi possível o isolamento de um único fator a influenciar estatisticamente na ocorrência de complicações, não podendo descartar uma participação das drogas na evolução menos satisfatória deste grupo.

A idade gestacional, no parto, foi significantemente menor no GHD do que no GHC. Além disso, 70,3\% dos prematuros eram do GHD, o que condiz com a literatura ${ }^{17}$. Por outro lado, Idris et al. ${ }^{15}$, analisando gestantes com hipertireoidismo tratado, não encontraram aumento da incidência de partos prematuros. No presente estudo, o único parâmetro que interferiu na idade gestacional foi o nível de T4L, no terceiro trimestre. De fato, estudos têm demonstrado que a tireotoxicose materna inadequadamente tratada está associada com risco aumentado de indicação de resolução da gravidez na fase pré-termo ${ }^{16}$, enquanto que o hipertireoidismo subclínico não se relaciona com a diminuição da idade gestacional no parto ${ }^{20}$. No presente estudo, fica patente a influência da gravidade da tireotoxicose, particularmente no final da gravidez, na evolução das gestantes, o que corrobora a necessidade do tratamento adequado conforme preconizado pela literatura ${ }^{22}$.

Com relação ao parto, as pacientes do GHD apresentaram uma alta taxa de cesáreas $(54,5 \%)$, levandose em consideração as pacientes do GHC $(35,5 \%)$ e a porcentagem de cesáreas do Serviço do Hospital das Clínicas da UNESP (42,8\%, dados não publicados), no período estudado. Algumas das principais indicações para este tipo de parto, e o caráter de urgência de muitas delas, sugerem a interferência do descontrole da tireotoxicose sobre este parâmetro de evolução gestacional, embora isto não tenha sido mostrado estatisticamente. O aumento da taxa de cesáreas em pacientes com hipertireoidismo tem sido relatado; recentemente, Idris et al. ${ }^{15}$, estudando gestantes com hipertireoidismo controlado na época do parto, relataram que a tireotoxicose materna, no início do seguimento, esteve associada com aumento da taxa de cesáreas $(33,3 \%)$, aumento este induzido pelos procedimentos de urgência. Por outro lado, quando os níveis de T4L se encontram dentro da faixa da normalidade, mesmo com os de TSH se mantendo supressos, não são relatados aumentos nas taxas de indicações de cesáreas ${ }^{20}$.

Conclui-se que o GHD recebeu doses maiores de medicamentos e apresentou resultados menos satisfatórios com relação à gestação, ao feto e parto do que o GHC. Exerceram influência sobre a evolução negativa das pacientes: o nível de T4L e a dose de PTU elevados, no terceiro trimestre, além da idade mais avançada de algumas gestantes. Assim, nas pacientes avaliadas, o hipertireoidismo descompensado exerceu influência sobre a morbidade materno-fetal. Contudo, há que se ressaltar que este estudo apresenta evidentes limitações, sendo que uma delas é o critério utilizado para classificação dos grupos, ou seja, os níveis séricos de T4L. Sabe-se que durante a gestação ocorrem alterações fisiológicas nas concentrações da tiroxina, provocadas, em parte, pelas alterações nos níveis da globulina ligadora de tiroxina ou TBG. Também são relatados níveis mais baixos de T4L em grávidas, quando comparadas a não-grávidas, e variações nas concentrações do hormônio no decorrer da gestação, o que sugere cautela na avaliação de resultados baseados neste parâmetro, indicando a necessidade de definição mais rigorosa de valores de referência para o estado gravídico $^{23}$. Outra limitação deste estudo é o seu caráter retrospectivo, baseado no levantamento de prontuários médicos, os quais, muitas vezes, mostram-se incompletos no seu preenchimento. Como a falta de dados costuma prejudicar a análise final dos resultados, estes devem ser considerados com cuidado.

\section{Agradecimentos}

Os autores agradecem à Fundação de Amparo à Pesquisa do Estado de São Paulo (FAPESP) pelo suporte financeiro (processo $n^{\circ}$. 05/52557-2), ao professor doutor José Eduardo Corrente, e Hélio Rubens de Carvalho Nunes, do Grupo de Apoio à Pesquisa da Faculdade de Medicina de Botucatu da UNESP, pelo auxílio na realização da análise estatística, e à professora doutora Izildinha Maestá, pelo fornecimento dos dados gerais sobre o movimento da Maternidade do Hospital das Clínicas da UNESP. 


\section{Referências}

1. Almeida CA, Vieira Neto L, Costa SM, Buescu A, Vaisman M. Hipertiroidismo por doença de Graves durante a gestação. Rev Bras Ginecol Obstet. 2005;27(5):263-7.

2. Glinoer D. Management of hypo- and hyperthyroidism during pregnancy. Growth Horm IGF Res. 2003;13 Suppl A:S45-54.

3. Greenspan FS, Strewler GJ. A glândula tireóide. In: Greenspan FS, Strewler GJ, editores. Endocrinologia básica \& clínica. 5th ed. Rio de Janeiro: Guanabara Koogan; 2000. p. 142-91.

4. Amino N, Izumi $Y$, Hidaka Y, Takeoka K, Nakata Y, Tatsumi KI, et al. No increase of blocking type anti-thyrotropin receptor antibodies during pregnancy in patients with Graves' disease. J Clin Endocrinol Metab. 2003;88(12):5871-4.

5. Roti E, Minelli R, Salvi M. Clinical review 80: Management of hyperthyroidism and hypothyroidism in the pregnant woman. J Clin Endocrinol Metab. 1996;81(5):1679-82.

6. Chan GW, Mandel SJ. Therapy insight: management of Graves' disease during pregnancy. Nat Clin Pract Endocrinol Metab. 2007;3(6):470-8.

7. De Castro JJ, Borges F. Hipertireoidismo e gravidez. Acta Med Port. 2003; 16(5):334-6.

8. Nachum Z, Rakover Y, Weiner E, Shalev E. Graves' disease in pregnancy: prospective evaluation of a selective invasive treatment protocol. Am J Obstet Gynecol. 2003;189(1):159-65.

9. Mestman JH. Hyperthyroidism in pregnancy. Best Pract Res Clin Endocrinol Metab. 2004;18(2):267-88.

10. Briceño Pérez C, Briceño Sanabria L. [Thyroid dysfunctions and pregnancy]. Ginecol Obstet Mex. 2006;74(9):462-70. Spanish.

11. Aoki Y, Belin RM, Clickner R, Jeffries R, Phillips L, Mahaffey KR. Serum TSH and total T4 in the United States population and their association with participant characteristics: National Health and Nutrition Examination Survey (NHANES 1999-2002). Thyroid. 2007; 17(12):1211-23.

12. Feki M, Omar S, Menif O, Tanfous NB, Slimane H, Zouari F, et al. Thyroid disorders in pregnancy: frequency and association with selected diseases and obstetrical complications in Tunisian women. Clin Biochem. 2008;41(12):927-31 .

13. Rodrigues LP, Jorge SRPF, Roveran V, Yamano LM, Vásquez ML, Aoki T, et al. Quantificação de anticorpos antitireoperoxidase e antitireoglobulina, tireotrofina e tiroxina livre em gestantes normais. Rev Bras Ginecol Obstet. 2007;29(9):478-83.

14. Prummel MF, Strieder T, Wiersinga WM. The environment and autoimmune thyroid diseases. Eur J Endocrinol. 2004; 150(5):605-18.

15. Idris I, Srinivasan R, Simm A, Page RC. Effects of maternal hyperthyroidism during early gestation on neonatal and obstetric outcome. Clin Endocrinol (Oxf). 2006;65(1):133-5.

16. Abalovich M, Amino N, Barbour LA, Cobin RH, De Groot L, Glinoer $D$, et al. Management of thyroid dysfunction during pregnancy and postpartum: an Endocrine Society Clinical Practice Guideline. J Clin Endocrinol Metab. 2007;92(8 Suppl):S1-47.

17. Atkins P, Cohen SB, Phillips BJ. Drug therapy for hyperthyroidism in pregnancy: safety issues for mother and fetus. Drug Saf. 2000;23(3):229-44.

18. Mandel SJ, Cooper DS. The use of antithyroid drugs in pregnancy and lactation. J Clin Endocrinol Metab. $2001 ; 86(6)$ :2354-9.

19. Nakagawa Y, Mori K, Hoshikawa S, Yamamoto M, Ito S, Yoshida K. Postpartum recurrence of Graves' hyperthyroidism can be prevented by the continuation of antithyroid drugs during pregnancy. Clin Endocrinol (Oxf). 2002;57(4):467-71.

20. Casey BM, Dashe JS, Wells CE, McIntire DD, Leveno KJ, Cunningham FG. Subclinical hyperthyroidism and pregnancy outcomes. Obstet Gynecol. 2006; 107(2 Pt 1):337-41.

21. Rezende J. Oligodrâmnio. In: Rezende J, editor. Obstetrícia. 9a ed. Rio de Janeiro: Guanabara Koogan; 2002. p. 878-81.

22. Cunha SP, Duarte G, Feitosa e Castro RM, Nogueira AA, Mauad Filho F, Debbs A. Hipertireoidismo e gravidez. Rev Bras Ginecol Obstet. 1996;18(3):243-7.

23. Vieira JGH, Kanashiro I, Tachibana TT, Ghiringhello MT, Hauache OM, Maciel RMB. Definição de valores normais de tiroxina livre durante a gravidez. Arq Bras Endocrinol Metab. 2004;48(2):305-9. 